\title{
Electrical Field Analysis and Optimizing of High Voltage Bushing Based on Maxwell
}

\author{
Zeng Linsuo \\ Electrical Engineering Department \\ SUT \\ Shenyang, China \\ zls1004@hotmail.com
}

\author{
Zhao Lei \\ Electrical Engineering Department \\ SUT \\ Shenyang, China \\ leiwuiai@sina.com
}

\begin{abstract}
In this paper, it was mainly discussed the electrical field analysis of the high voltage outlet in the insulation structure, which was a part of the transformers manufacture. Firstly, the electrical field of $500 \mathrm{kV}$ transformers-outlet devices inside the enclosure was modeled in Maxwell electrostatics solution type and calculated under the industrial frequency withstands voltage with Finite Element Method. The calculation was used to analyze the intensity of electric field about the oil gaps and that around the equalizing ring surface. Then, a model simplification was made and the shape parameters were defined. Further optimizations from the Maxwell optimetrics department were used to search for the optimal solutions. At last, the solutions were reused in the former model and we could contrast the safety factor of the oil gaps and the intensity of the equalizing ring. The result turned out to be effective to optimize the high voltage outlet in the insulation structure.
\end{abstract}

Keywords-outlet insulation structure; FEM; electrical-field calculations; Maxwell;optimizing

\section{INTRODUCTION}

Since Weidmann from Switzerland studied and developed the manufacturing technology of lead insulation moldings of in 1960s, it has been attracting attention of scholars at home and abroad that the calculation for electrical field of lead and bushing on ultra-high voltage power transformers, especially the part inside enclosure, which is narrow, small and complex with a plunge from working electric potential to zero within a short distance. This paper analyzes the outlet insulation structure of a HV bushing within oil of a power transformer typed SFP10$400 \mathrm{MVA} / 500 \mathrm{KV}$. The electric field analyzed results will provide theoretical foundations for the design of transformer-outlet insulation structure and theoretical parameters for the R\&D of other projects.

\section{BUILDING FEM MODELING}

\section{A. Simplified calculated modeling}

The HV bushing modeling for calculation in this paper is shown in Fig .1. It is installed that an equalizing tube outside the lead to optimize the electrode, and that an equalizing ring under the HV bushing to improve the electrical-field around. There are insulating pulps and molding insulation paper tubes attached to the equalizing ring and equalizing tube, in order to separate the oil gaps and improve the electric intensity. It is filled with transformer oil among the enclosure ${ }^{[1,2]}$.

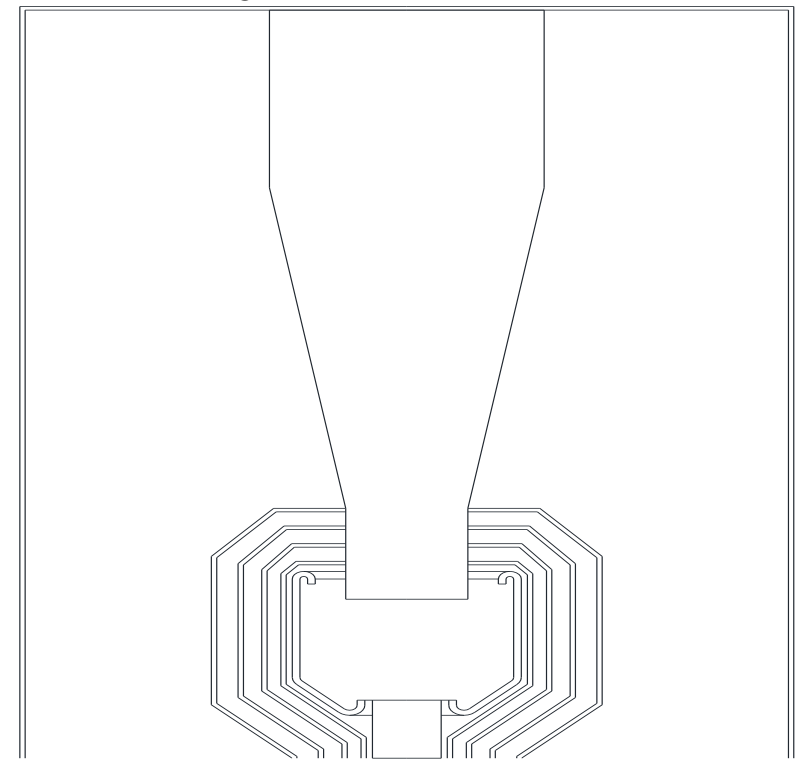

Figure 1. HV bushing model within oil

Before the electrical-field calculation, to be easy to handle, it is necessary to simply the actual field and the domain properly and assume as follows:

- Ignoring the interference between coil and lead.

- Assuming that the space in the enclosure is filled with oil totally, without any impurities like air gaps or welding slag, etc.

- Ignoring the effect of metal or woody clamping structures around lead.

- Assuming the domain of HV bushing within oil is completely symmetry centering the lead.

So that, it may analyze 2-dimesion finite field, provided we build a 1/2 shaft section with Ansys Maxwell, which leads to a combination of simplification of analytical procedure and time, and benefit of subsequent parametric analysis and electrical-field optimizations.

\section{B. Setting the boundary conditons}

For the $500 \mathrm{kV}$ power transformers, the most rigor test conditions in design assess of insulation level is industrial frequency withstands voltage $680 \mathrm{kV}$, which ought to be the high potential in analysis. And the electric field 
calculation under industrial frequency can be approximated to quasi-static field calculation ${ }^{[3]}$. The material of enclosure chooses steel_1008, given zero electric potential; the equalizing ring and equalizing tube are connected equipotentially, the material of which chooses copper, given $1 \mathrm{~min}$ industrial frequency withstand voltage $680 \mathrm{kV}$ on the surface; the material of bushing chooses ceramics, given linear voltage from bottom $680 \mathrm{kV}$ to top flange $0 \mathrm{~V}$, above are on the first boundary condition. The partial differentials of electric potential of the left boundary and the down one to normal vector are zero, which are on the second boundary condition. The dielectric constants of kinds of mediums are as follows: transformer oil $\varepsilon_{1}=2.2$, insulation paper tubes $\varepsilon_{2}=4.5$, NOMEX insulating pulps $\varepsilon_{3}=1.6$, ceramics $\varepsilon_{4}=7.0$.

The differential equation for the electric calculation is $\nabla^{2} \varphi=0$. The boundary value problem involved above may conclude as ${ }^{[4-7]}$ :

$$
\left\{\begin{array}{l}
\frac{1}{r} \frac{\partial}{\partial r}\left(r \frac{\partial \varphi}{\partial r}\right)+\frac{\partial^{2} \varphi}{\partial z^{2}}=0, \\
\varphi_{1}=680 \mathrm{kV}, \\
\varphi_{2}=0, \\
\frac{\partial \varphi}{\partial n}=0, \\
\varepsilon_{a} \frac{\partial \varphi}{\partial n}=\varepsilon_{b} \frac{\partial \varphi}{\partial n} .
\end{array}\right.
$$

\section{COMPUTATION AND ANALYSIS}

In oil-paper insulation structure, the insulating property mainly depends on the electric intensity of the transformer oil. As the electric field intensity born by oil and insulating cardboard is inversely proportional to the dielectric constants, the one in oil gaps is much higher. So once the oil gaps had a breakdown, the insulating cardboard would lose the insulation ability. Therefore, this paper mainly researches the insulating strength of oil gaps. According to the small volume effect theory of electric intensity of oil gaps, Weidmann from Switzerland proposed the allowable curve for insulation design of transformer, which has been providing the basis for design without partial discharge. The allowable curve for insulation design when the initial probability value of partial discharge equals $1 \%$ may be concluded as:

$$
E=K * d^{-0.37}
$$

In the formula, $K$ is a constant related to the quality of transformer oil and the position of oil gaps; $d$ expresses the length of oil gaps along the power line, in degree $\mathrm{mm}$. $E$ expresses the allowable values of the initial strength of partial discharge, maximum of $11 \mathrm{kV} \cdot \mathrm{mm}^{-1[8-9]}$.

It is evidently that the maximum of electric field intensity around the equalizing ring present to the first oil gap according to the Maxwell FEM calculation. The electric field intensity of oil gaps is calculated along the power line stating at maximum point of field intensity. It is contrasted with the curve of (2), which leads to Fig .3. And the variation of electric field strength along the equalizing ring edge is shown in Fig .4.
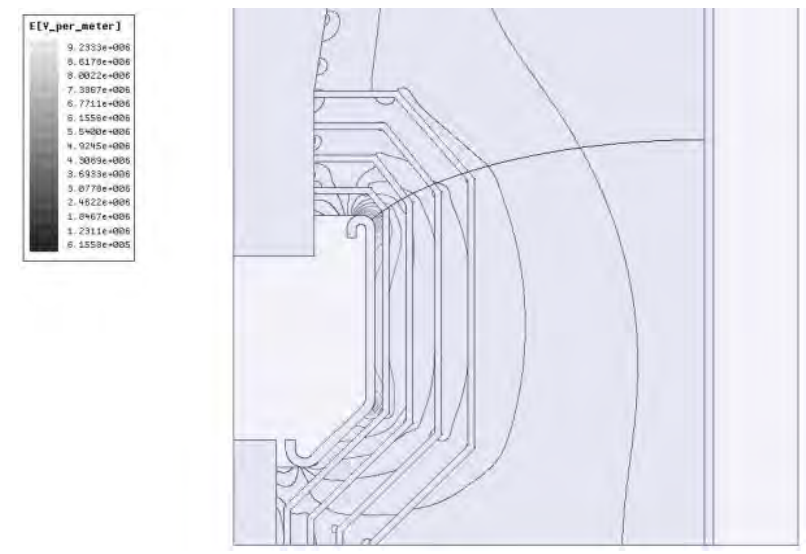

Figure 2. Field intensity around the equalizing ring

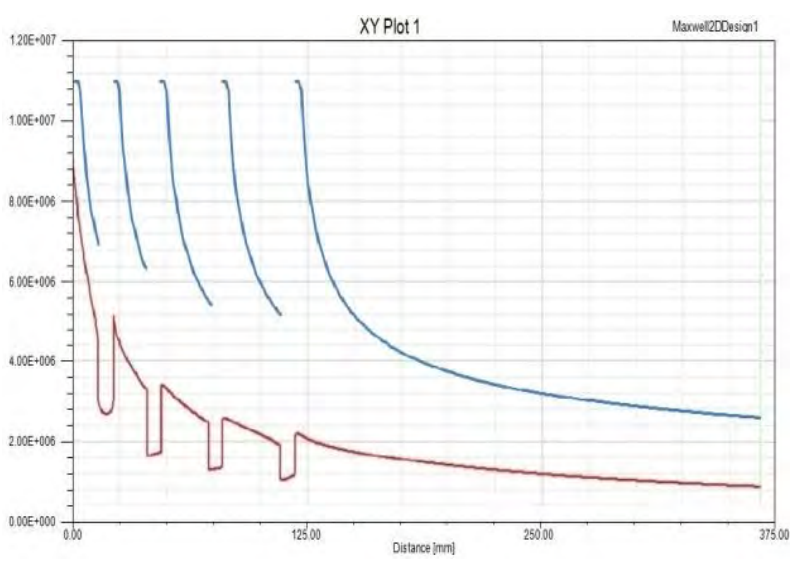

Figure 3. Contrast between field intensity of oil gaps along the power line and maximun

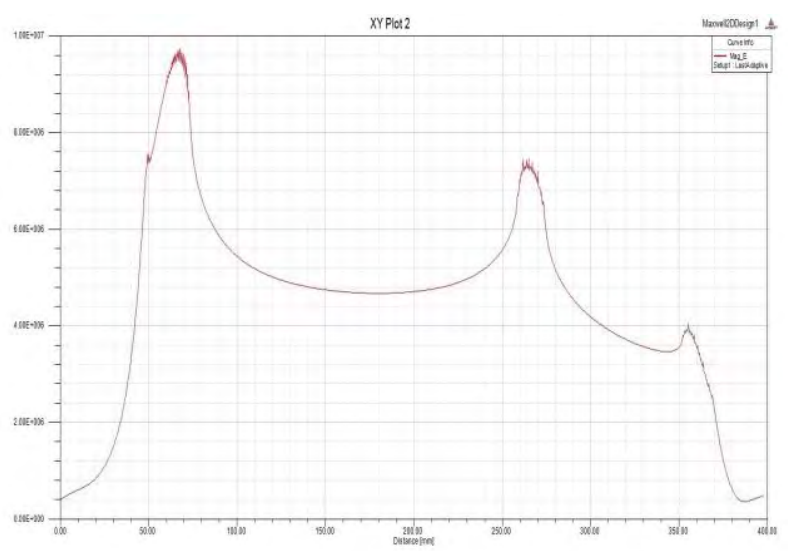

Figure 4. Distribution of electric field intensity along the equalizing ring edge

It is evidently in Fig . 3 that the field intensity in each oil gap and the one around the equalizing ring surface are under the maximum. However, in the first oil gap, the values are close to some extent, lowering the safety factor. And along the equalizing ring edge, three circular arcs lead to three jumps, and the whole field intensity fluctuates widely. Now the external radiuses of three circular arcs are $15 \mathrm{~mm}, 20 \mathrm{~mm}, 20 \mathrm{~mm}$ respectively. It can be considered to optimize the whole shape to promote the safety factors of oil gaps and stabilize the distribution of electric field intensity along the equalizing ring edge. 


\section{OPTIMIZATION PROCEDURE}

\section{A. Conversion of Equivalent Dielectric Constants}

It requests for fairly meticulous finite element subdivision that the paper oil insulation part calculating the electric field. And shape optimizations would make subdivisions for this part repeatedly, which leads to huge workload. Thus, to decrease the quantity of elements, this paper equivalents multilayer paper oil insulation into some single medium in the method of equivalent dielectric constants. It ought to be invariant that the shape, dimension and the electric field energy of electrode before optimizations; it ought to be invariant that the electric field intensity above the surface of electrode. According to the analytic formula of electric field distribution in coaxial cylindrical capacitor medium of layer $i$

$$
E=\frac{U}{r \varepsilon_{\mathrm{i}} \sum_{\mathrm{i}=1}^{\mathrm{n}} \frac{1}{\varepsilon_{\mathrm{i}}} \ln \frac{r_{\mathrm{i}}}{r_{\mathrm{i}-1}}},
$$

we can get

$$
\sum_{i=1}^{n} \frac{1}{\varepsilon_{i}} \ln \frac{r_{i}}{r_{i}-1}=\frac{1}{\varepsilon} \ln \frac{r_{n}}{r_{1}}
$$

The equivalence of paper oil insulation in this paper results $\varepsilon=2.52$.

\section{B. Optimization Algorithms}

The peak of field intensity above the equalizing ring surface depends major on the circular arcs' radiuses, minor on lines' length and other factors. Therefore, in the optimization procedure of paper oil insulation structure around the equalizing ring, circular arcs' radiuses are optimization variables. A mathematic model is made as follow:

$$
\min f(X), X=\left(x_{1}, x_{2}, \ldots, x_{n}\right)^{T}
$$

$f(X)$ is objective function, the peak of field intensity above the equalizing ring; $X$ is design variable, the external radiuses of three circular arcs above the equalizing ring surface respectively ${ }^{[10-11]}$.

Maxwell optimetrics department offers five optimization algorithms, Sequential Nonlinear Programming (SNLP), Sequential Mixed Integer Nonlinear Programming (SMINLP), Quasi Newton, Pattern Search and Genetic Algorithm. They may all achieve satisfactory conclusions based on analysis. After making a simple model, the first external radius of circular arc above the equalizing ring surface is set as $r l$, the second one is set as $r 2$, and the third one is the same to the second one for the limit of shape. So that, there are two parameters in the whole calculation model, and the optimization target are minimum peaks of field intensity around the circular arcs. Considering the actual manufacturing technology, $r 1$ is defined from $10 \mathrm{~mm}$ to $25 \mathrm{~mm}, r 2$ from $15 \mathrm{~mm}$ to $25 \mathrm{~mm}$.

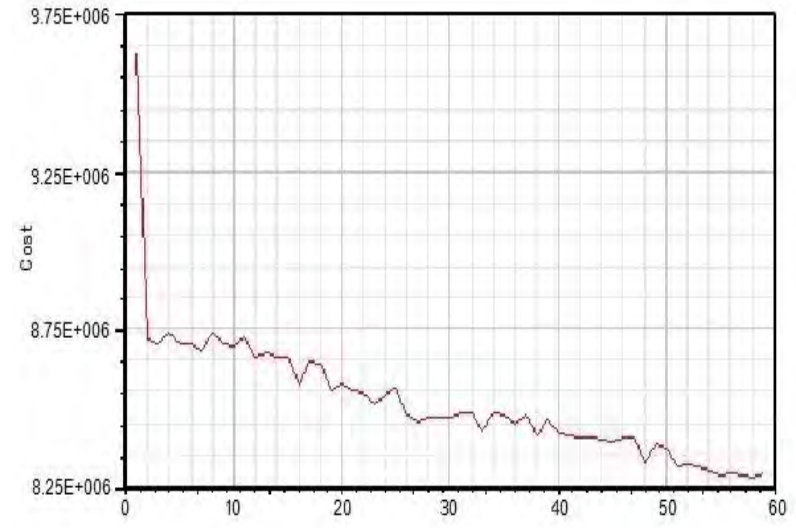

Figure 5a. Iteration times of $\mathrm{r} 1$ and field intensity variation

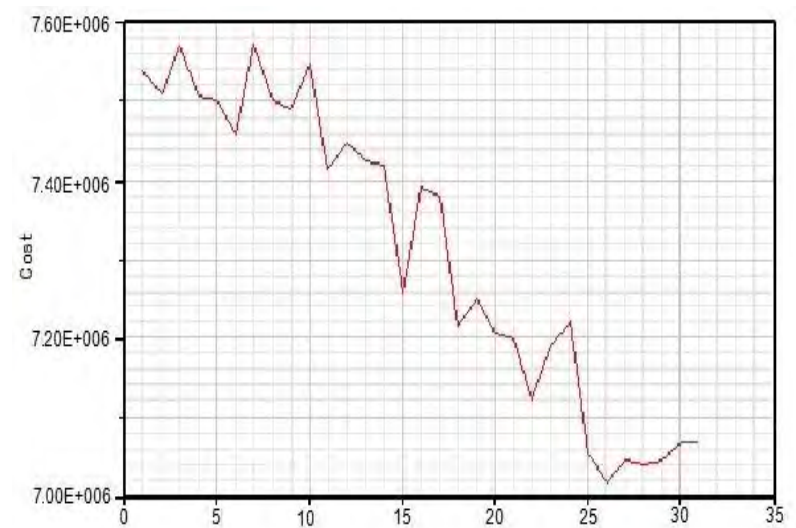

Figure $5 \mathrm{~b}$. Iteration times of $\mathrm{r} 2$ and field intensity variation

We may optimize $r 1$ and $r 2$ with Quasi Newton respectively: $r l$ achieves the optimal value at $58^{\text {th }}$ iteration and $r 2$ achieves at $26^{\text {th }}$. Right now, $r l=25.00 \mathrm{~mm}$, $r 2=22.70 \mathrm{~mm}$. Adjusting three external radiuses of circular arcs in Fig .2 to new dimensions, and recalculating the electric field intensity of oil gaps, we can get Fig .6; Contrasting the distribution of electric field intensity along the equalizing ring edge with Fig .4, we can get Fig .7. It shows that the optimizations have an ideal effect.

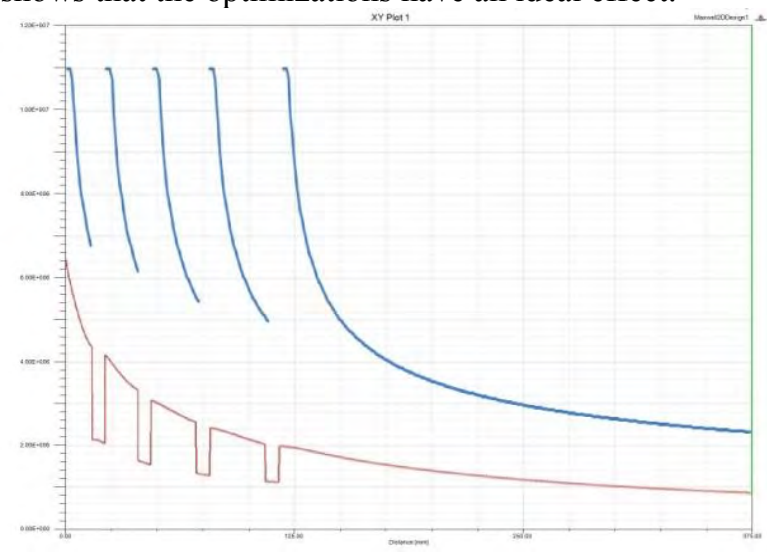

Figure 6. Contrast between field intensity of oil gaps along the power line and maximun after optimizations 


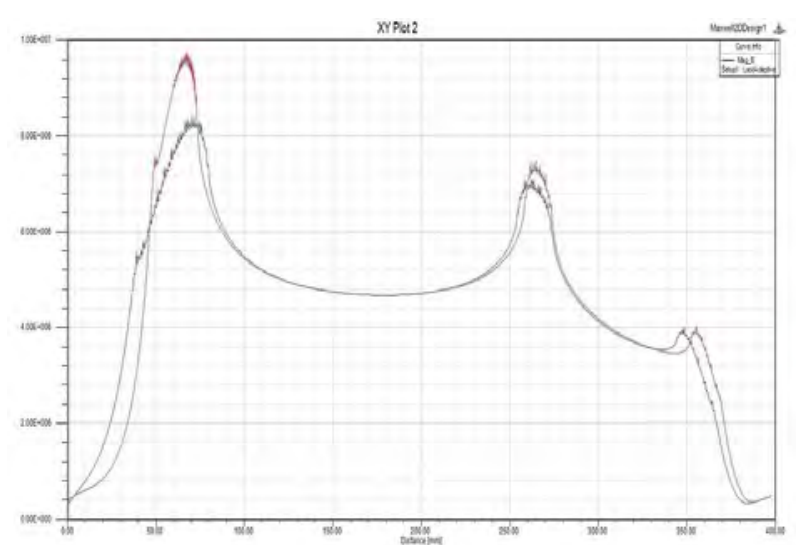

Figure 7. Contrast of distribution of electric field intensity along the equalizing ring edge

\section{CONCLUSION}

We calculated the electric field intensity of $\mathrm{HV}$ bushing model in $500 \mathrm{kV}$ UHV power transformer enclosure with FEM in Maxwell. It displayed the distribution of each oil gap, which indicated a low safety factor after contrast with the allowed values. Also, the electric field intensity around the equalizing ring surface fluctuated widely. We respectively optimized three external radiuses of circular arcs with Quasi Newton in Maxwell optimetrics. We could find that the safety factors increased with the field intensity of oil gaps decreased, and the waves above the equalizing ring stabilized. However, it is not definitely obvious optimizing with two parameters merely. It is expected to continue the optimization with the factors such as changing the enclosure diameter or others.

\section{REFERENCES}

[1] MI Chuanlong, XIE Qingfeng, MENG Likun, HAN Xiaodong. "Design and Application of Exits Insulation Structure for EHV and UHV AC Transformers," High Voltage Engineering, vol. 36, No. 1, Jan. 31, 2010, pp. 122-128.

[2] WANG Shaojun, CHEN Xinfu and MENG Likun, "A New Type of UHV Indirect Outlet Structure," High Voltage Apparatus, vol. 49, No. 12, Dec.2013, pp.150-154

[3] YAN Xiuke, SUN Yang, YU Cunzhan, XIE Diexin. "Electric Field Analysis and Optimal Design of Main Insulation for Oil-Immersed Inverted Current Transformers," Transactions of China Electrotechnical Society, vol. 29, No. 1, Jan. 2014, pp. 37-43.

[4] LIU Peng, FENG Yong, ZHANG Shiling, PENG Zhongren. "Optimization for Ultra-High Voltage AC Bushing Screening Electrode Structure," Journal of Xi'an Jiaotong University, vol. 46, No. 2, Feb. 2012, pp. 88-92.

[5] ZHANG Xile, WANG Jianmin, WU Zengbo, QI Guifen, SUN Youliang, CHEN Zhiwei. "Numerical Analysis of Electric Field in Valve Side Lead-Out Wire for UHV DC Converter Transformer," Transformer, vol.46, No.7, July. 2009, pp. 1-4.

[6] YAN Weili, Yang Qinxin, Electrical Engineering Electromagnetic Field Numerical Analysis. Beijing, China: China Machine Press, 2005.

[7] GAO Youhua, DING Chunhong, WANG Guogang. "Optimization and Analysis of Main Insulation Structure in Power Transformer," Transformer, vol.46, No.10, Oct. 2009, pp. 1-5.

[8] ZHAO Feng, LU Tiebing, WANG Bing. "Application of Finite Element Method in Insulation Design of Ultra-High Voltage Transformer," Insulating Meterials, vol.46, No.1, 2013, pp. 66-69.

[9] LUO Qinglin, ZHONG Juntao. "Insulation Design of UHV Transformer to Reduce PD Probability," Transformer, vol. 48, No. 11, Nov. 2011, pp. 16-18.

[10] HANG Chenhui, SHI Peifeng, BAO Jinchun. "Major Insulation Field Calculation of UHV Transformer," Electric Switchgear, No. 6, 2011, pp. 50-51. 